\title{
Zenith Wet Delay Retrieval Using Two Different Techniques for the South American Region and Their Comparison
}

\author{
A. Calori, G. Colosimo, M. Crespi, F. Azpilicueta, M. Gende, C. Brunini, \\ and M.V. Mackern
}

\begin{abstract}
Retrieving atmospheric water vapor content using GNSS claimed the attention of the geodetic community ever since the beginning of the GPS deployment. The main purpose of the present work is to propose a comparison of the Zenith Wet Delay (ZWD) retrieved by GPS with the direct measurements provided by the water vapor radiometer loaded on-board the Jason-1 altimetry satellite and those obtained from SIRGAS (Geocentric Reference System for the Americas) GNSS reference stations. In this respect, the work proposes a methodology for the comparison and contributes to assess the capabilities of SIRGAS permanent network to provide water vapor informations that can be useful both for shortterm weather forecasting and for long-term climate studies. For the period from June 2008 to June 2010 the tropospheric parameters of more than 100 SIRGAS stations were estimated using Bernese 5.0 software with a time interval of $15 \mathrm{~min}$. Since Jason-1 returns reliable measurements only over open ocean areas, a subset of 14 stations located along the coastline was selected for the comparison. A dedicated software was developed in order to effectively manage the huge amount of Jason-1 data, mainly devoted to data selection according to site position, time interval and data filtering using quality flag indicators. The Zenith Hydrostatic Delay (ZHD) provided by the European Center for Medium Weather Forecasting (ECMWF) were first corrected up to the GPS station height and then used to derive the ZWD from the GPS estimated Zenith Total Delay (ZTD). The agreement between the techniques was evaluated in terms of bias and standard deviation of the differences (i.e. $\mathrm{GPS}_{Z W D}-$ Jason- $1_{Z W D}$ ) resulting in $7.4 \mathrm{~mm}$ and $15.4 \mathrm{~mm}$, respectively. The average correlation coefficient is 0.93 .
\end{abstract}

\section{Keywords}

Zenith wet delay retrieval • GNSS • Jason-1 radiometer • SIRGAS permanent network

A. Calori • M.V. Mackern

Facultad de Ingeniería, Universidad Nacional de Cuyo, Mendoza, Argentina

G. Colosimo $(\bowtie) \cdot$ M. Crespi

DICEA-Area di Geodesia e Geomatica, University of Rome

"La Sapienza", Rome, Italy

e-mail: gabriele.colosimo@uniroma1.it

M. Gende • C. Brunini • F. Azpilicueta

Facultad de Ciencias Astronómicas, Universidad Nacional de La Plata, La Plata, Argentina

\section{Introduction}

Water vapor has been recognized as the most important greenhouse gas (Mitchell 1989). Improving the capacity to measure its spatial distribution and its temporal variability would constitute a great advance for understanding a variety of weather processes with relevant influence on human welfare and environmental care (Perler et al. 2011). Radiosondes and microwave radiometers, either located on the ground or 
loaded on-board low Earth orbiters, are routinely used to provide direct measurements of this parameter. Nonetheless, these techniques are still far from providing global coverage and short-time resolution and remain rather expensive. In this context, the possibility of estimating the ZWD and then the Integrated Water Vapor (IWV) content from dense networks of continuously operating Global Navigation Satellite Systems (GNSS) reference stations claimed the attention of the geodetic community ever since the beginning of the Global Positioning System (GPS) era (e.g. Bevis et al. 1992). In fact, retrieving meteorological information from processing ground based GNSS network data is in continuous development (i.e. Rocken et al. 1995; Duan et al. 1996; Tregoning et al. 1998; Buyn and Bar-Sever 2009; Bonafoni et al. 2012).

Given these understandings, it appears clear that SIRGAS GNSS permanent network, apart from being the regional densification of the International Terrestrial Reference Frame (ITRF) in an extensive and heterogeneous area that encloses American and Antarctica continents, can be thought of as an important test field towards climate change studying and comprehension. SIRGAS network, in fact, spans a huge extension $20^{\circ}>\phi>-65^{\circ},-12^{\circ}>\lambda>-65^{\circ}$, with altitudes up to $3.770 \mathrm{~m}$ and, at present, it encompasses about 250 continuously operating GNSS reference stations, 48 of them belongs to International GNSS Service (IGS) network. Exploiting SIRGAS network, previous studies aimed to investigate and compare GPS and other techniques (e.g. radiosondes and radiometers) as regards the water vapor retrieval (e.g. Sapucci et al. (2007) using three permanent stations located over Amazonian region and considering a period of 3 months, Fernández et al. (2010) using four SIRGAS permanent stations and considering a 1 year period).

This work joins the previous ones and aims to perform a comparison between the ZWD retrieved by GPS observations in 14 sites homogeneously distributed along the South American coastline (belongs to one of the various SIRGAS sub networks SIRGAS-CON-D-SUR) with the direct measurements provided by the water vapor radiometer on-board the Jason-1 altimetry satellite (i.e. Jason-1 Microwave Radiometer (JMR)). The comparison was carried out in terms of ZWD over a time period of 2 years (i.e. from June 2008 to June 2010). This research is a part of a wider study aiming to assess the suitability of GNSS for producing water vapor maps in such a large region that encompasses many different climate conditions as SIRGAS. All the differences between the two independent techniques, such as the spatial and temporal resolution, were addressed in order to yield a reliable comparison in terms of tropospheric ZWD.

Section 2 describes the main features of the used techniques and summarizes some capabilities of the software implemented to manage the huge amount of Jason-1 data. The results achieved are discussed in Sect. 3. Section 4 draws some conclusions and focuses onto further aspects to be investigated in the future.

\section{$2 \quad$ Adopted Techniques}

\section{$2.1 \quad$ GNSS}

Globally, the presence of Earth's atmosphere increases the optical path length between GNSS satellites and receivers and the corresponding travel time of the GNSS signals. A common praxis is to unfold the overall tropospheric delay into two separate components. A first larger delay, which can reach up to $2.3 \mathrm{~m}$ in the zenith direction, is due to the hydrostatic gases and is commonly referred to as ZHD. Due to its slight spatial and temporal variability the ZHD is effectively modeled with a few millimeters accuracy; in this analysis, the a priori hydrostatic model of Saastamoinen (1973) is used. On the other hand, the second component is spawned directly from the water vapor and is referred to as the ZWD. Although the ZWD contributes to a much smaller extent to the total delay, it displays a large temporal and spatial variability and, as a consequence, is rather difficult to model and predict. The tropospheric ZTD is the sum of the ZHD and the ZWD

$$
Z T D=Z H D+Z W D
$$

It is noteworthy that the longer the ray path along the troposphere the higher the caused delay will be. This effect is taken into account by using so called mapping function that describes the delay dependence on the zenith angle of the satellite. Normally, different mapping functions are adopted for the hydrostatic and the wet delay (Dach et al. 2007, p. 242).

SIRGAS Continuously Operating Network (CON) consists of more than 200 GNSS stations which are grouped into four sub-networks: a continental one with approximately 100 stations distributed over South America and the Caribbean (SIRGAS-CON-C), and three densification subnetworks covering the northern, middle and the southern part, respectively, of the SIRGAS region (Mackern et al. 2009). These networks are weekly computed by several processing centers and the individual solutions are combined by the IGS Regional Network Associate Analysis Centre for SIRGAS (IGS RNAAC SIR).

Within this research work, the tropospheric zenith parameters were estimated for approximately 100 GNSS SIRGAS permanent stations (SIRGAS-CON-D-SUR) using Bernese GPS Software 5.0 (Dach et al. 2007). GPS pseudorange and carrier phase data, provided in daily RINEX files with an acquisition rate of $30 \mathrm{~s}$, were utilized as input observations together with IGS precise products (i.e. satellite orbits and clocks, Earth orientation parameters) and the IGS absolute 


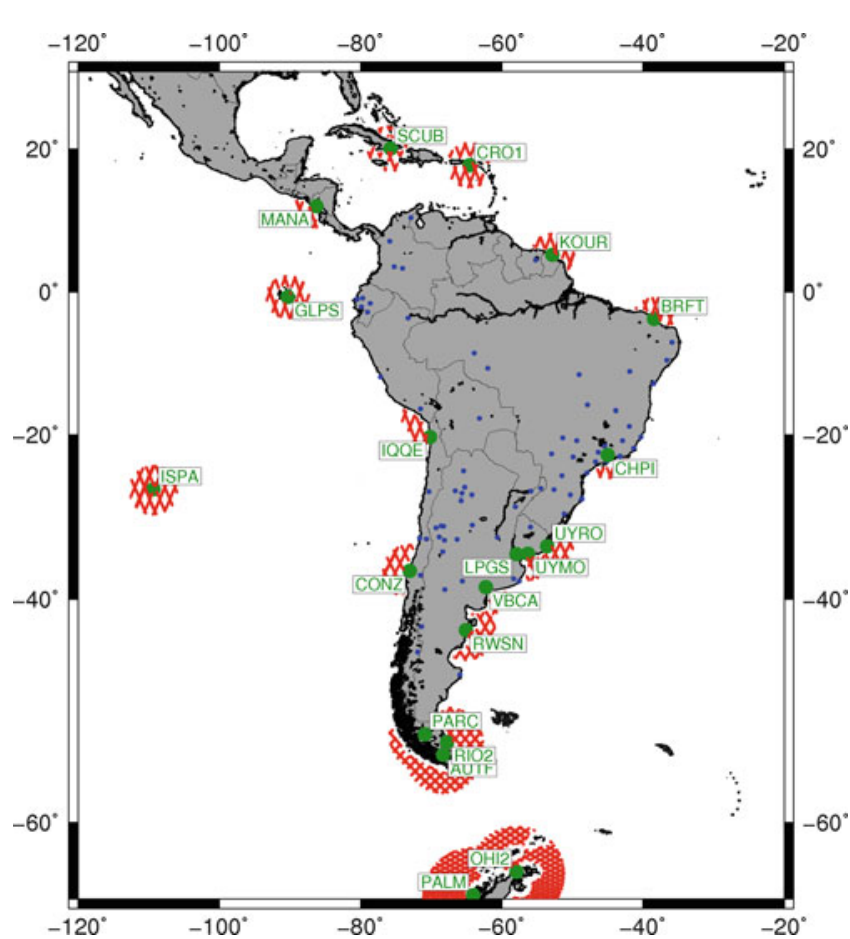

Fig. 1 Overall distribution of the approximately 100 stations of SIRGAS-CON-D-SUR network processed within this work (displayed in blue). The subset of 20 stations used in the comparison with Jason-1 radiometer, selected from the whole set according to two geographical criteria of limiting the coastline distance and the station height, are displayed in green (with greater dots). Red pattern displays the results of the automatic procedure implemented in order to select Jason-1 measurements that lie inside a circular area of a certain radius centered on stations selected by the user. Here, an area with radius of $1^{\circ}$ is displayed

calibration values for satellites and receivers antenna phase center corrections. Tropospheric ZTD were estimated every 15 min using the a priori hydrostatic model of Saastamoinen (1973) and the hydrostatic and wet Niell (1996) mapping functions. An elevation mask of $3^{\circ}$ was applied to the observations whereas the horizontal tropospheric gradients were not considered in the estimation process. Permanent stations coordinates were constrained to the weekly solutions provided by SIRGAS itself [repeatability at the level of $2 \mathrm{~mm}$ for the horizontal components and $4 \mathrm{~mm}$ in the height (Brunini et al. 2012)].

Overall, GPS contribution to the comparison consists of site-specific troposphere parameters estimated every $15 \mathrm{~min}$ (with a global formal accuracy at the level of $1 \mathrm{~mm}$ ) for approximately 100 stations within a global period of 2 years, from June 2008 to June 2010. An overview of station distribution over South American region is displayed in Fig. 1.

\subsection{Jason-1 and Satellite Radiometry}

Jason-1 is an oceanography mission launched December 7, 2001 and jointly operated by the Centre National d'Etudes
Spatiales (CNES) (i.e. the French aerospace agency) and the United States National Aeronautics and Space Administration (NASA). Jason-1 primarily aims to study the global circulation of the ocean and to reveal how the Earth's heat moves around through ocean currents.

The main instrument loaded on-board the spacecraft is the Dual-frequency Solid State Radar Altimeter (Poseidon-2) that is used to retrieve ocean topography with an accuracy of a few centimeters (Picot et al. 2003). Jason-1 is also equipped with ancillary sensors that either account for measurement delays or are used to precisely determine the satellite orbit (e.g. GPS receiver, laser retroreflector array, Dual frequency Doppler Orbitography and Radiopositioning by Satellite (DORIS) receiver). The delay caused by the water vapor along the altimeter beam is one of the most critical term to deal with in order to achieve the highest accuracy in determining the sea level topography. Consequently, Jason-1 is equipped with a refined radiometer that measures the brightness temperatures at three different frequencies (i.e. $18.7,23.8$ and $34.0 \mathrm{GHz}$ ) in the nadir direction over a circular footprint approximately between 20 and $30 \mathrm{~km}$. The $23.8 \mathrm{GHz}$ channel is devoted to water vapor measurement whereas the 18.7 and $34.0 \mathrm{GHz}$ channels provide the correction for the wind induced effects in the sea surface background emissions and the correction for cloud liquid water, respectively. Moreover, it is worth recalling that the radiometer measurements are severely degraded on the land because of the complications in determining the temperature of the hot background. Hence, the brightness temperatures are combined to yield the delay error caused by the water vapor in the atmosphere (i.e. the ZWD) according to a wellknown algorithm (described in Kehim et al. 1995, p. 155) with a Root Mean Square Error (RMSE) of $1.2 \mathrm{~cm}$ that is, however, limited to open ocean areas (Ruf et al. 1994).

Jason-1 is a low orbiting satellite (i.e. altitude $1336 \mathrm{~km}$ ) with a repeating period of approximately 10 days and its products are available to the users through different services (e.g. standard file transfer protocol maintained by agencies such as AVISO or PO.DAAC, the Radar Altimeter Database System (RADS)) either in near real-time (i.e. by means of the Operational Sensor Data Records, with a latency of 3-5h) or with an higher latency.

For the sake of the present work, we have chosen to utilize the Geophysical Data Records (GDR): a fully validated product that uses precise satellite orbits, applies dedicated ground retracking and has a latency of 30 days. GDR contain all the measurements collected from the satellite payloads as well as other ancillary informations that are required in order to obtain the sea surface height. This latter set encompasses, among others, the hydrostatic meteorological correction (ZHD) provided by the ECMWF and necessary to obtain the ZTD due to the troposphere (Eq. (1)). In more details, the ECMWF models the ZHD as being 
dependent on the surface pressure and, to a smaller extent, on latitude $\phi$

$$
Z H D_{E C M W F}=-2.277 \cdot P_{a t m}(1+0.0026 \cdot \cos 2 \phi)
$$

where the ZHD is returned in millimeters and the atmospheric pressure $\left(P_{\text {atm }}\right)$ is expressed in mbar and is obtained from ECMWF data with an accuracy of few mbar (Picot et al. 2003, p. 50). Moreover, the ECMWF model enriches GDR with a value of ZWD that is meant to backup the radiometer measurements in case either land contamination or unexpected behaviors affect the accuracy of the instrument. For either cases, an interpolation is required to refer ECMWF corrections at the time and location of the altimeter's measurement. Quality flags are included in the GDR to indicate the effectiveness of this interpolation and the data quality.

\subsection{Jason-1 Measurements Handling and Data Comparison Methodology}

Given the main features of the utilized techniques, this section describes how Jason-1 measurements were handled in order to be duly compared with the GPS estimates. Jason-1 GDR data are organized into PASS files, where each PASS is half a revolution of the Earth by the satellite (e.g. approximately 3,400 records with an acquisition interval of $1 \mathrm{~s}$ ). The passes are numbered from 1 to 254 representing a full repeat cycle (i.e. $\sim 10$ days) of Jason-1 ground track.

For the sake of the present work, 75 Jason-1 cycles (i.e. from cycle $\mathrm{C} 235$ to $\mathrm{C} 310$ ) of GDR version c binary data, corresponding to the period from June 2008 to June 2010, were downloaded from the web. Then, a tuned software was implemented to efficiently manage this large amount of information (i.e. more than 400 Gbytes after binary to ASCII conversion) and to select the data of interest for the comparison. In particular, given a (GPS site) position and a time interval selected by the user, the software explores the whole Jason-1 dataset and returns only the measurements collected within a circular area centered on the station itself. Additionally, the software takes care of filtering the data in order to retain only ocean observations and to remove any bad, missing, or flagged measurements (as recommended in Picot et al. 2003, pp. 13-14). After the bad data remotion step, the accuracies are compliant with the Jason-1 target values (Picot et al. 2003, p. 28).

As mentioned, radiometer returns reliable measurements only over open ocean areas; therefore, only the GNSS sites located in the proximity of the coastlines could be effectively used. Hence, a subset of 20 stations was selected from the bundle of GPS sites according to the two geographical criteria of limiting the distance from coastline and the station height (i.e. the closer the station is to the sea, the more similar will be the climate conditions with respect to those at the sea level). Figure 1 shows the distribution of the selected stations (green dots) subset and the Jason-1 ground tracks (red lines) for cycle 275 (i.e. $\sim 10$ days of measurements).

Exploiting the capabilities of the described implemented software, Jason-1 measurements were singled out choosing a circular radius of $1^{\circ}(\sim 100 \mathrm{~km})$ for each of the selected stations. The effectiveness of this procedure can be appreciated by Fig. 1: from the whole Jason-1 measurements, originally covering all the ocean surface, only those in the proximity of the selected GPS sites were retained, thereby dramatically decreasing the time required for the further comparison.

Some important differences between the radiometer measurements and the GNSS estimates should be addressed to yield a reliable comparison. Firstly, JMR measures the ZWD whereas GNSS estimates the total delay due to the troposphere (i.e. the ZTD). Hence, as it is reported in Eq. (1), the ZHD provides the link between the two techniques. In this research, since we were lacking pressure observations for most of the used sites, and given the high accuracy claimed by the ECMWF model (at a few mbar level, Picot et al. 2003, p. 50), it has been decided to use the ZHD values provided in the GDR data in order to face Jason-1 and GPS in terms of ZWD. In more details, following Haines and Bar-Sever (1998) and Desai and Haines (2004), the $Z^{2 W D}{ }_{G P S}$ at the antenna height $(h)$ was derived as follows

$$
Z W D_{G P S}(h)=Z T D_{G P S}(h)-Z H D_{E C M W F}(h)
$$

Secondly, GPS estimations are referred to the station height whereas the radiometer refers its measurements at sea level. In this regard, consistently with Kouba (2008), we decided to address this height difference separately for the dry and the wet components. For the dry component, we used the pressure value at the sea level $\left(P_{a t m}\right)$, inferred by inverting Eq. (2), to correct the $Z H D_{E C M W F}$ up to the GPS antenna height $(h)$ above the sea level, using Davis et al. (1985) approximation for the $\mathrm{ZHD}(\mathrm{h})$

$$
Z \operatorname{HD}_{E C M W F}(h)=a \frac{P(h)}{(1-b \cdot \cos (2 \phi)-c \cdot h)}
$$

and Berg (1948) standard pressure model

$$
P(h)=P_{a t m}(1-d \cdot h)^{5.225}
$$

where $a=0.0022768, b=0.00266, c=0.28 \cdot 10^{-6}$ and $d=0.0000226$. For the JMR measurement, since no convenient transformation is available, we applied the following empirical formula proposed by Kouba (2008)

$$
Z W D_{J M R}(h)=Z W D(0) \cdot e^{-(h) / 2000}
$$




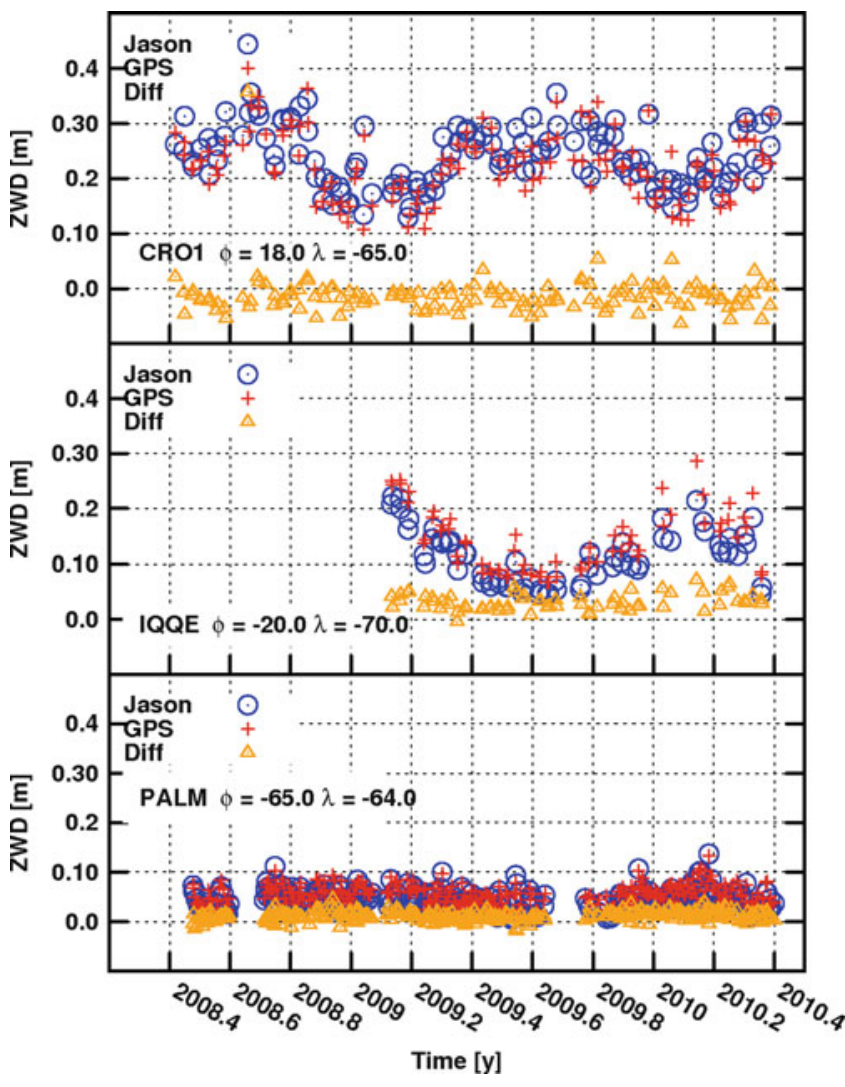

Fig. 2 Time series of the JMR measurements (blue circles) and the GPS estimations (red crosses) for three of the analyzed stations (i.e. CRO1, IQQE, PALM). The ZWD differences (i.e. $\mathrm{GPS}_{Z W D}-\mathrm{JMR}_{Z W D}$ ) are displayed in orange

Finally, the $Z W D_{G P S}(h)$ values, derived from the original $15 \mathrm{~min}$ estimates using Eq. (3), were linearly interpolated across the time series to obtain the value at times of Jason-1 overflight.

\section{Results and Discussion}

As explained, the comparison between ZWD retrieved by GPS and JMR was referred to GPS permanent station height. Figure 2 shows the time series for three sample stations (i.e. CROI, IQQE and PALM). The GPS values are reported with red crosses whereas JMR ones are shown using blue circles. Analyzing the time series of the differences, reported in Fig. 2 with orange stars, neither linear drifts nor annual behavior are highlighted. The discontinuities in the time series arise either because of GPS observations lack or because the station was not considered in the SIRGASCON-D-SUR network processing (e.g. the first period of IQQE station).

The agreement between the techniques was evaluated using the bias $(\mu)$, the standard deviation $(\sigma)$ of the differences (i.e. $G P S_{Z W D}-\mathrm{JMR}_{Z W D}$ ) as statistical indexes.
Table 1 Biases, standard deviations and correlations of the differences (i.e. $\mathrm{GPS}_{Z W D}-\mathrm{JMR}_{Z W D}$ ) for 14 stations

\begin{tabular}{lrrrrrl}
\hline Name & $\mathrm{h}(\mathrm{m})$ & Dist $(\mathrm{km})$ & Num \# & $\mu(\mathrm{mm})$ & $\sigma(\mathrm{mm})$ & \multicolumn{1}{l}{$\rho$} \\
\hline BRFT & 22 & 6 & 31 & 1.4 & 20.9 & 0.90 \\
\hline CONZ & 181 & 26 & 39 & 10.2 & 14.2 & 0.91 \\
\hline CRO1 & -32 & 1 & 133 & -16.0 & 20.9 & 0.93 \\
\hline GLPS & 2 & 0 & 38 & -0.2 & 10.1 & 0.98 \\
\hline IQQE & 39 & 0 & 66 & 31.1 & 14.9 & 0.97 \\
\hline ISPA & 112 & 13 & 51 & 5.1 & 18.2 & 0.91 \\
\hline MANA & 71 & 45 & 49 & 4.3 & 17.6 & 0.96 \\
\hline OHI2 & 33 & 0 & 80 & 10.4 & 8.8 & 0.87 \\
\hline PALM & 31 & 0 & 314 & 10.8 & 9.5 & 0.89 \\
\hline RIO2 & 32 & 4 & 63 & 9.0 & 9.9 & 0.94 \\
\hline RWSN & 24 & 6 & 28 & 11.8 & 8.2 & 0.94 \\
\hline SCUB & 22 & 6 & 64 & -9.5 & 29.0 & 0.89 \\
\hline UYMO & 158 & 2 & 53 & 14.7 & 15.8 & 0.95 \\
\hline UYRO & 59 & 2 & 43 & 20.0 & 16.9 & 0.97 \\
\hline Average values & & 1,052 & 7.4 & 15.4 & 0.93 \\
\hline
\end{tabular}

Fourth column reports the amount of comparison per station

Additionally, the correlation $(\rho)$ of the time series was evaluated. Unfortunately, a consistent number of JMR measurements covers only 14 out of 20 GNSS stations close enough to the coastline and previously selected; for this reason, only 14 stations were considered. The results for these stations are reported in Table 1.

The fifth column indicates, with a positive average bias of $7.4 \mathrm{~mm}$, that generally GPS overestimates ZWD with respect to Jason-1. However, for most of the station this bias is not significant (i.e. the average $\sigma$ of the differences is $15.4 \mathrm{~mm}$ ). With no sake of deriving a correlation between the bias and the latitude, it can be interesting to note that the only negative biases are displayed by the two northern stations (i.e. $C R O 1$ and $S C U B$ ), whereas the stations located souther than $30^{\circ}$ have a positive bias greater than $8 \mathrm{~mm}$. Generally speaking, the order of magnitude of the bias is consistent with the results obtained by previous and important inter-technique comparison campaigns (e.g. Desai and Haines 2004; Edwards et al. 2004; Bock et al. 2010; Teke et al. 2011). This testifies that the inter-technique differences were correctly accounted for. The correlations coefficient is always higher than 0.87 and reaches an average value of 0.93 without displaying any dependence on the latitude. The impact of station height and distance from coastline is not clearly distinguishable by the results. However, more investigations are necessary in order to get a better comprehension.

\section{Conclusions and Perspectives}

ZWD obtained by JMR radiometer were compared with those retrieved by 14 stations of the SIRGAS GNSS permanent network. The primary aim of this comparison was to investigate the agreement of the techniques in such a 
broad region as South and Central America. The statistical indexes used to assess the comparison (i.e. bias and standard deviation of the differences $G P S_{Z W D}-\mathrm{JMR}_{Z W D}$ and correlation of the time series) resulted in average values of $7.4 \mathrm{~mm}, 15.4 \mathrm{~mm}$ and $0.93 \mathrm{~mm}$, respectively. These values are in accordance with results of previous researches. On one hand this testifies that the inter-technique differences were correctly accounted for, on the other it further confirms SIRGAS capabilities to contribute to short and long term meteorological studies.

Further aspects to refine the comparison include addressing the spatial difference between GPS sites and Jason-1 measurements (e.g. extrapolate JMR measurements to the point of closest station approach), considering the contribution of other constellations (e.g. Glonass) and enlarging the comparison to other regions (i.e. with a dense GNSS network nearby the coastline) to possibly investigate the latitude dependency.

Acknowledgements We thank all the Reviewers for their valuable suggestions that helped improving the present research. This work was partially supported by Progetto di cooperazione Scientifica $e$ Tecnologica Italia-Argentina 2011-2013.

\section{References}

Berg H (1948) Allgemeine meteorologie. Duemmler, Bonn

Bevis M, Businger S, Herring TA, Rocken C, Anthes RA, Ware RH (1992) GPS meteorology: remote sensing of the atmospheric water vapor using the Global Positioning System. J Geophys Res 97:15787-15801

Bock O, Willis P, Lacarra M, Bosser P (2010) An inter-comparison of zenith tropospheric delays derived from DORIS and GPS data. Adv Space Res 46(10):1648-1660

Bonafoni S, Mazzoni A, Cimini D, Montopoli M et al (2012) Assessment of water vapor retrievals from a GPS receiver network. GPS Solut. doi:10.1007/s10291-012-0293-5

Brunini C, Sánchez L, Drewes H, Costa S, Mackern V, Martínez W et al (2012) Improved analysis strategy and accessibility of the SIRGAS reference frame. In: IAG symposia, 2012, vol 136, Part 1, pp 3-10

Buyn S, Bar-Sever Y (2009) A new type of troposphere zenith path delay product of the International GNSS service. J Geodes 83:367373. doi:10.1007/s00190-008-0288-8

Dach R, Hugentobler H, Fridez P, Meindl M (2007) Bernese GPS Software Version 5.0, User manual. Astronomical Institute, University of Bern, Bern

Davis JL, Herring TA, Shapiro II et al (1985) Geodesy by radio interferometry: effects of atmospheric modeling errors on estimates of baseline length. Radio Sci 20(6):1593-1607
Desai SD, Haines BJ (2004) Monitoring measurements from the Jason-1 microwave radiometer and independent validation with GPS. Mar Geodes 27:221-240

Duan J, Bevis M, Fang P, Bock Y, Chiswell S et al (1996) GPS meteorology: direct estimation of the absolute value of precipitable water. J Appl Meteorol 35:830-838

Edwards S, Moore P, King M (2004) Assessment of the Jason-1 and TOPEX/Poseidon microwave radiometer performance using GPS from offshore sites in the North sea. Mar Geodes 27: $717-727$

Fernández LI, Salio P, Natali MP, Meza AM (2010) Estimation of precipitable water vapor from GPS measurements in Argentina. Validation and quantitative analysis of results. Adv Space Res 46:879-894

Haines BJ, Bar-Sever Y (1998) Monitoring the TOPEX microwave radiometer with GPS: stability of columnar water vapor measurements. Geo Res Lett 25:3563-3566

Kehim S, Janssen M, Ruf C (1995) TOPEX/Poseidon microwave radiometer (TMR): III. Wet troposphere range correction algorithm and pre-launch error budget. IEEE Trans Geosci Remote Sens 33(1):147-161

Kouba J (2008) Implementing and testing of the gridded Vienna Mapping Function 1 (VMF1). J Geodes 82:193-205

Mackern MV, Mateo ML, Robin AM, Calori AV (2009) A terrestrial reference frame, coordinates and velocities for South American stations: contributions to Central Andes geodynamics. Adv Geosci 22:181-184

Mitchell JFB (1989) The greenhouse effect and climate change. Rev Geophys 27:115-139

Niell AE (1996) Global mapping functions for the atmosphere delay at radio wavelengths. J Geophys Res 101:3227-3246

Perler D, Geiger A, Hurter F (2011) 4D GPS water vapor tomography: new parameterized approaches. J Geodes 85:539-550. doi:10.1007/s00190-011-0454-2

Picot N, Case K, Desai S, Vincent P (2003) AVISO and PODAAC user handbook. IGDR and GDR Jason Products, SMM-MU-M5-OP-13184-CN (AVISO), JPL D-21352 (PODAAC)

Rocken C, Van Hove T, Johnson J, Solheim F et al (1995) GPS/STORM: GPS sensing of atmospheric water vapor for meteorology. J Atmos Ocean Technol 12:468-478

Ruf CS, Keihm SJ, Subramanya B, Janssen MA (1994) TOPEX/POSEIDON microwave radiometer performance and in-flight calibration. J Geophys Res 99:24915-24926

Saastamoinen II (1973) Contribution to the theory of atmospheric refraction. Bull Géodésique 107:13-34

Sapucci LF, Machado LAT, Monico JFG, Plana-Fattori A (2007) Intercomparison of Integrated Water Vapor estimates from multisensors in the Amazonian region. J Atmos Ocean Technol 24:1880-1894

Teke K, Böhm, Nilsson T, Schuh H, Steigenberger P, Dach R, Heinckelmann R, Willis P, Haas R et al (2011) Multi-technique comparison of troposphere zenith delays and gradients during CONT08. J Geodes 85:395-413

Tregoning P, Boers R, O Brien D (1998) Accuracy of absolute precipitable water vapor estimates from GPS observations. J Geophys Res 103:28701-28710 